\title{
Convergence of three-step iterations for Ciric-quasi contractive operator in $\mathrm{CAT}(0)$ spaces
}

\author{
Gurucharan S. Saluja \\ Department of Mathematics, \\ Govt. Nagarjuna P.G. College of Science, \\ Raipur - 492001 (C.G.), India. \\ email: saluja1963@gmail.com
}

\begin{abstract}
In this paper, we study three-step iteration process for Ciricquasi contractive operator and establish strong convergence theorems for above mentioned operator and schemes in the setting of CAT $(\mathbf{0})$ spaces. Our result extends and generalizes some previous work from the existing literature (see, e.g., $[4,30]$ and some others).
\end{abstract}

\section{Introduction}

A metric space $\mathrm{X}$ is a $\mathrm{CAT}(0)$ space if it is geodesically connected and if every geodesic triangle in $\mathrm{X}$ is at least as 'thin' as its comparison triangle in the Euclidean plane. It is well known that any complete, simply connected Riemannian manifold having non-positive sectional curvature is a CAT(0) space. For a thorough discussion of these spaces and of the fundamental role they play in geometry, we refer the reader to Bridson and Haefliger [8].

Fixed point theory in CAT(0) spaces was first studied by Kirk (see [22, 23]). He showed that every nonexpansive (single-valued) mapping defined on a bounded closed convex subset of a complete CAT(0) space always has a fixed

2010 Mathematics Subject Classification: 54H25, 54E40.

Key words and phrases: Ciric-quasi contractive operator, three-step iteration scheme, fixed point, strong convergence, $\mathrm{CAT}(0)$ space. 
point. Since, then the fixed point theory for various mappings and iteration schemes in a CAT(0) space has been rapidly developed and a lot of papers appeared (see, [3, 11, 13, 14, 20, 21, 24, 25, 27, 31, 32]). It is worth mentioning that the results in $\mathrm{CAT}(0)$ spaces can be applied to any $\mathrm{CAT}(\mathrm{k})$ space with $k \leq 0$ since any $\operatorname{CAT}(k)$ space is a $\operatorname{CAT}\left(k^{\prime}\right)$ space for every $k^{\prime} \geq k$ (see,e.g., $[8])$.

Let $(X, d)$ be a metric space. A geodesic path joining $x \in X$ to $y \in X$ (or, more briefly, a geodesic from $x$ to $y$ ) is a map $c$ from a closed interval $[0, l] \subset \mathbb{R}$ to $X$ such that $c(0)=x, c(l)=y$, and let $d\left(c(t), c\left(t^{\prime}\right)\right)=\left|t-t^{\prime}\right|$ for all $t, t^{\prime} \in[0, l]$. In particular, $c$ is an isometry, and $d(x, y)=l$. The image $\alpha$ of $c$ is called a geodesic (or metric) segment joining $x$ and $y$. We say $X$ is (i) a geodesic space if any two points of $\mathrm{X}$ are joined by a geodesic and (ii) uniquely geodesic if there is exactly one geodesic joining $x$ and $y$ for each $x, y \in X$, which we will denoted by $[x, y]$, called the segment joining $x$ to $y$.

A geodesic triangle $\triangle\left(x_{1}, x_{2}, x_{3}\right)$ in a geodesic metric space $(X, d)$ consists of three points in $X$ (the vertices of $\triangle$ ) and a geodesic segment between each pair of vertices (the edges of $\triangle$ ). A comparison triangle for geodesic triangle $\triangle\left(x_{1}, x_{2}, x_{3}\right)$ in $(X, d)$ is a triangle $\bar{\triangle}\left(x_{1}, x_{2}, x_{3}\right):=\triangle\left(\overline{x_{1}}, \overline{x_{2}}, \overline{x_{3}}\right)$ in $\mathbb{R}^{2}$ such that $d_{\mathbb{R}^{2}}\left(\overline{x_{i}}, \overline{x_{j}}\right)=d\left(x_{i}, x_{j}\right)$ for $i, j \in\{1,2,3\}$. Such a triangle always exists (see [8]).

\section{CAT(0) space}

A geodesic metric space is said to be a CAT(0) space if all geodesic triangles of appropriate size satisfy the following $\mathrm{CAT}(0)$ comparison axiom.

Let $\triangle$ be a geodesic triangle in $X$, and let $\triangle \subset \mathbb{R}^{2}$ be a comparison triangle for $\triangle$. Then $\triangle$ is said to satisfy the $\operatorname{CAT}(0)$ inequality if for all $x, y \in \triangle$ and all comparison points $\bar{x}, \bar{y} \in \bar{\triangle}$,

$$
d(x, y) \leq d_{\mathbb{R}^{2}}(\bar{x}, \bar{y}) .
$$

Complete CAT(0) spaces are often called Hadamard spaces (see [19]). If $x, y_{1}, y_{2}$ are points of a $\operatorname{CAT}(0)$ space and $y_{0}$ is the mid point of the segment $\left[y_{1}, y_{2}\right]$ which we will denote by $\left(y_{1} \oplus y_{2}\right) / 2$, then the CAT(0) inequality implies

$$
d^{2}\left(x, \frac{y_{1} \oplus y_{2}}{2}\right) \leq \frac{1}{2} d^{2}\left(x, y_{1}\right)+\frac{1}{2} d^{2}\left(x, y_{2}\right)-\frac{1}{4} d^{2}\left(y_{1}, y_{2}\right)
$$

The inequality (2) is the ( $\mathrm{CN}$ ) inequality of Bruhat and Tits [9].

Let us recall that a geodesic metric space is a CAT(0) space if and only if it satisfies the $(\mathrm{CN})$ inequality (see [[8], p.163]). Moreover, if $\mathrm{X}$ is a CAT(0) 
metric space and $x, y \in X$, then for any $\alpha \in[0,1]$, there exists a unique point $\alpha x \oplus(1-\alpha) y \in[x, y]$ such that

$$
\mathrm{d}(z, \alpha x \oplus(1-\alpha) \mathrm{y}) \leq \alpha \mathrm{d}(z, x)+(1-\alpha) \mathrm{d}(z, y),
$$

for any $z \in X$ and $[x, y]=\{\alpha x \oplus(1-\alpha) y: \alpha \in[0,1]\}$.

A subset $C$ of a $C A T(0)$ space $X$ is convex if for any $x, y \in C$, we have $[x, y] \subset C$.

Algorithm 1. The sequence $\left\{x_{n}\right\}$ defined by $x_{1} \in C$ and

$$
x_{n+1}=a_{n} T x_{n}+\left(1-a_{n}\right) x_{n}, \quad n \geq 1,
$$

where $\left\{a_{n}\right\}_{n=1}^{\infty}$ is a sequence in $(0,1)$ is called a Mann iterative sequence (see $[26])$.

Algorithm 2. The sequence $\left\{x_{n}\right\}$ defined by $x_{1} \in C$ and

$$
\begin{aligned}
y_{n} & =b_{n} T x_{n}+\left(1-b_{n}\right) x_{n}, \\
x_{n+1} & =a_{n} T y_{n}+\left(1-a_{n}\right) x_{n}, \quad n \geq 1,
\end{aligned}
$$

where $\left\{a_{\mathfrak{n}}\right\}_{\mathfrak{n}=1}^{\infty}$ and $\left\{b_{\mathfrak{n}}\right\}_{\mathfrak{n}=1}^{\infty}$ are appropriate sequences in $[0,1]$ is called an Ishikawa iterative sequence (see [17]).

Algorithm 3. The sequence $\left\{x_{n}\right\}$ defined by $x_{1} \in C$ and

$$
\begin{aligned}
z_{n} & =c_{n} T x_{n}+\left(1-c_{n}\right) x_{n}, \\
y_{n} & =b_{n} T z_{n}+\left(1-b_{n}\right) x_{n}, \\
x_{n+1} & =a_{n} T y_{n}+\left(1-a_{n}\right) x_{n}, \quad n \geq 1,
\end{aligned}
$$

where $\left\{\mathbf{a}_{\mathfrak{n}}\right\}_{\mathfrak{n}=1}^{\infty},\left\{\mathbf{b}_{\mathfrak{n}}\right\}_{\mathfrak{n}=1}^{\infty},\left\{\mathbf{c}_{\mathfrak{n}}\right\}_{\mathfrak{n}=1}^{\infty}$ are appropriate sequences in $(0,1)$ is called Noor iterative sequence (see [28]).

Algorithm 4. The sequence $\left\{x_{n}\right\}$ defined by $x_{1} \in C$ and

$$
\begin{aligned}
y_{n} & =b_{n} T x_{n}+\left(1-b_{n}\right) x_{n}, \\
x_{n+1} & =a_{n} T y_{n}+\left(1-a_{n}\right) T x_{n}, \quad n \geq 1,
\end{aligned}
$$

where $\left\{a_{n}\right\}_{\mathfrak{n}=1}^{\infty}$ and $\left\{b_{n}\right\}_{n=1}^{\infty}$ are appropriate sequences in $(0,1)$ is called $S$ iterative sequence (see [2]).

Recently, Abbas and Nazir [1] introduced the following iterative process: 
Algorithm 5. The sequence $\left\{x_{n}\right\}$ defined by $x_{1} \in C$ and

$$
\begin{aligned}
x_{n+1} & =\left(1-a_{n}\right) T y_{n}+a_{n} T z_{n}, \\
y_{n} & =\left(1-b_{n}\right) T x_{n}+b_{n} T z_{n}, \\
z_{n} & =\left(1-c_{n}\right) x_{n}+c_{n} T x_{n}, n \geq 1
\end{aligned}
$$

where $\left\{a_{n}\right\},\left\{b_{n}\right\}$ and $\left\{c_{n}\right\}$ are sequences in $(0,1)$. They showed that this process converges faster than the Agarwal et al. [2].

Very recently, Thakur et al. [33] introduced the following iterative process:

Algorithm 6. The sequence $\left\{x_{n}\right\}$ defined by $x_{1} \in C$ and

$$
\begin{aligned}
x_{n+1} & =\left(1-a_{n}\right) T x_{n}+a_{n} T y_{n}, \\
y_{n} & =\left(1-b_{n}\right) z_{n}+b_{n} T z_{n}, \\
z_{n} & =\left(1-c_{n}\right) x_{n}+c_{n} T x_{n}, n \geq 1
\end{aligned}
$$

where $\left\{a_{n}\right\},\left\{b_{n}\right\}$ and $\left\{c_{n}\right\}$ are sequences in $(0,1)$. They showed that this process converges faster than all of the Picard, the Mann, the Ishikawa, the Noor, the Agarwal et al. and the Abbas et al. processes for contractions in the sense of Berinde [5] and in support gave analytic proof by a numerical example.

We now modify (9) in a $\operatorname{CAT}(0)$ space as follows.

Let $\mathrm{C}$ be a nonempty closed convex subset of a complete CAT(0) space $X$ and $\mathrm{T}: \mathrm{C} \rightarrow \mathrm{C}$ be a mapping. Suppose that $\left\{x_{n}\right\}$ is a sequence generated iteratively by

$$
\begin{aligned}
x_{n+1} & =\left(1-a_{n}\right) T x_{n} \oplus a_{n} T y_{n}, \\
y_{n} & =\left(1-b_{n}\right) z_{n} \oplus b_{n} T z_{n}, \\
z_{n} & =\left(1-c_{n}\right) x_{n} \oplus c_{n} T x_{n}, n \geq 1
\end{aligned}
$$

where $\left\{a_{n}\right\},\left\{b_{n}\right\}$ and $\left\{c_{n}\right\}$ are sequences in $(0,1)$.

If we put $c_{n}=0$ for all $n \geq 1$, then (10) reduces to the following iteration process

$$
\begin{aligned}
x_{n+1} & =\left(1-a_{n}\right) T x_{n} \oplus a_{n} T y_{n}, \\
y_{n} & =\left(1-b_{n}\right) x_{n} \oplus b_{n} T x_{n},
\end{aligned}
$$

where $\left\{a_{n}\right\}$ and $\left\{b_{n}\right\}$ are sequences in $(0,1)$ is called modified S-iteration process.

We recall the following. 
Let $(X, d)$ be a metric space and $\mathrm{T}: X \rightarrow X$ be a mapping. A mapping $\mathrm{T}: \mathrm{X} \rightarrow \mathrm{X}$ is called an a-contraction if

$$
d(T x, T y) \leq \operatorname{ad}(x, y)
$$

where $a \in(0,1)$ and for all $x, y \in X$.

The mapping $T$ is called Kannan mapping [18] if there exists $b \in\left(0, \frac{1}{2}\right)$ such that

$$
d(T x, T y) \leq b[d(x, T x)+d(y, T y)]
$$

for all $x, y \in X$.

The mapping $T$ is called Chatterjea mapping [12] if there exists $c \in\left(0, \frac{1}{2}\right)$ such that

$$
d(T x, T y) \leq c[d(x, T y)+d(y, T x)]
$$

for all $x, y \in X$.

In 1972, combining these three definitions, Zamfirescu [34] proved the following important result.

Theorem $\mathbf{Z}$. Let $(X, d)$ be a complete metric space and $T: X \rightarrow X$ a mapping for which there exists the real number $a, b$ and $c$ satisfying $a \in(0,1), b, c \in$ $\left(0, \frac{1}{2}\right)$ such that for any pair $x, y \in X$, at least one of the following conditions holds:

$$
\begin{aligned}
& \left(z_{1}\right) d(T x, T y) \leq a d(x, y), \\
& \left(z_{2}\right) d(T x, T y) \leq b[d(x, T x)+d(y, T y)], \\
& \left(z_{3}\right) d(T x, T y) \leq c[d(x, T y)+d(y, T x)] .
\end{aligned}
$$

Then $T$ has a unique fixed point $p$ and the Picard iteration $\left\{x_{\mathfrak{n}}\right\}_{\mathfrak{n}=0}^{\infty}$ defined by $x_{n+1}=T x_{n}, n=0,1,2, \ldots$ converges to $p$ for any arbitrary but fixed $x_{0} \in X$.

The conditions $\left(z_{1}\right)-\left(z_{3}\right)$ can be written in the following equivalent form

$$
d(T x, T y) \leq h \max \left\{d(x, y), \frac{d(x, T x)+d(y, T y)}{2},\right.
$$


for all $x, y \in X$ and $0<h<1$, has been obtained by Ciric [10] in 1974 .

A mapping satisfying (15) is called Ciric quasi-contraction. It is obvious that each of the conditions $\left(z_{1}\right)-\left(z_{3}\right)$ implies (15).

An operator T satisfying the contractive conditions $\left(z_{1}\right)-\left(z_{3}\right)$ in the theorem $\mathrm{Z}$ is called Z-operator.

In 2000, Berinde [4] introduced a new class of operators on a normed space E satisfying

$$
\|\mathrm{T} x-\mathrm{T} y\| \leq \delta\|x-y\|+\mathrm{L}\|\mathrm{T} x-x\|, \quad(*)
$$

for any $x, y \in E, 0 \leq \delta<1$ and $L \geq 0$.

He proved that this class is wider than the class of Zamfirescu operators and used the Mann iteration process to approximate fixed points of this class of operators in a normed space given in the form of following theorem.

Theorem B. Let $C$ be a nonempty closed convex subset of a normed space E. Let T: C $\rightarrow C$ be an operator satisfying $(*)$. Let $\left\{x_{n}\right\}_{n=0}^{\infty}$ be defined by: for $x_{1}=x \in C$, the sequence $\left\{x_{n}\right\}_{n=0}^{\infty}$ given by $(5)$ where $\left\{a_{n}\right\}$ is a sequence in $[0,1]$. If $\mathrm{F}(\mathrm{T}) \neq \emptyset$ and $\sum_{n=1}^{\infty} a_{n}=\infty$, then $\left\{x_{n}\right\}_{n=0}^{\infty}$ converges strongly to the unique fixed point of $\mathrm{T}$.

In this paper, inspired and motivated by [33, 34], we study an iteration process (10) and establish strong convergence theorems to approximate the fixed point for Ciric quasi contractive operator in the framework of CAT(0) spaces.

We need the following useful lemmas to prove our main result in this paper.

Lemma 1 (See [27]) Let $\mathrm{X}$ be a CAT(0) space.

(i) For $\mathrm{x}, \mathrm{y} \in \mathrm{X}$ and $\mathrm{t} \in[0,1]$, there exists a unique point $z \in[\mathrm{x}, \mathrm{y}]$ such that

$$
\mathrm{d}(x, z)=\mathrm{td}(x, y) \quad \text { and } \quad \mathrm{d}(\mathrm{y}, z)=(1-\mathrm{t}) \mathrm{d}(\mathrm{x}, \mathrm{y}) .
$$

We use the notation $(1-t) x \oplus$ ty for the unique point $z$ satisfying $(A)$.

(ii) For $\mathrm{x}, \mathrm{y} \in \mathrm{X}$ and $\mathrm{t} \in[0,1]$, we have

$$
d((1-t) x \oplus t y, z) \leq(1-t) d(x, z)+t d(y, z) .
$$

Lemma 2 (See [6]) Let $\left\{\mathrm{p}_{\mathfrak{n}}\right\}_{\mathfrak{n}=0}^{\infty},\left\{\mathbf{q}_{\mathfrak{n}}\right\}_{\mathfrak{n}=0}^{\infty},\left\{\boldsymbol{r}_{\mathfrak{n}}\right\}_{\mathfrak{n}=0}^{\infty}$ be sequences of nonnegative numbers satisfying the following condition:

$$
\mathrm{p}_{\mathrm{n}+1} \leq\left(1-\mathrm{s}_{\mathrm{n}}\right) \mathrm{p}_{\mathrm{n}}+\mathrm{q}_{\mathrm{n}}+\mathrm{r}_{\mathrm{n}}, \forall \mathrm{n} \geq 0,
$$

where $\left\{s_{n}\right\}_{n=0}^{\infty} \subset[0,1]$. If $\sum_{n=0}^{\infty} s_{n}=\infty, \lim _{n \rightarrow \infty} q_{n}=\mathrm{O}\left(s_{n}\right)$ and $\sum_{n=0}^{\infty} r_{n}<$ $\infty$, then $\lim _{n \rightarrow \infty} p_{n}=0$. 


\section{Strong convergence theorems in CAT(0) Space}

In this section, we establish strong convergence result of iteration process (10) to approximate a fixed point for Ciric quasi contractive operator in the framework of $\mathrm{CAT}(0)$ spaces.

Theorem 1 Let $\mathrm{C}$ be a nonempty closed convex subset of a complete CAT(0) space $\mathrm{X}$ and let $\mathrm{T}: \mathrm{C} \rightarrow \mathrm{C}$ be an operator satisfying the condition (15). Let $\left\{x_{n}\right\}$ be defined by the iteration scheme (10). If $\sum_{n=1}^{\infty} a_{n} b_{n}=\infty$, then $\left\{x_{n}\right\}$ converges strongly to the unique fixed point of $\mathrm{T}$.

Proof. By Theorem Z, we know that $\mathrm{T}$ has a unique fixed point in $\mathrm{C}$, say $\mathbf{u}$. Consider $x, y \in C$. Since $T$ is a operator satisfying (15), then if

$$
\begin{aligned}
d(T x, T y) & \leq \frac{h}{2}[d(x, T x)+d(y, T y)] \\
& \leq \frac{h}{2}[d(x, T x)+d(y, x)+d(x, T x)+d(T x, T y)]
\end{aligned}
$$

implies

$$
\left(1-\frac{h}{2}\right) d(T x, T y) \leq \frac{h}{2} d(x, y)+h d(x, T x),
$$

which yields (using the fact that $0<\mathrm{h}<1$ )

$$
d(T x, T y) \leq\left(\frac{h / 2}{1-h / 2}\right) d(x, y)+\left(\frac{h}{1-h / 2}\right) d(x, T x) .
$$

If

$$
\begin{aligned}
d(T x, T y) & \leq \frac{h}{2}[d(x, T y)+d(y, T x)] \\
& \leq \frac{h}{2}[d(x, T x)+d(T x, T y)+d(y, x)+d(x, T x)]
\end{aligned}
$$

implies

$$
\left(1-\frac{h}{2}\right) d(T x, T y) \leq \frac{h}{2} d(x, y)+h d(x, T x),
$$

which also yields (using the fact that $0<\mathrm{h}<1$ )

$$
d(T x, T y) \leq\left(\frac{h / 2}{1-h / 2}\right) d(x, y)+\left(\frac{h}{1-h / 2}\right) d(x, T x) .
$$

Denote

$$
\mathrm{L}_{1}=\max \left\{\mathrm{h}, \frac{\mathrm{h} / 2}{1-\mathrm{h} / 2}\right\}=\mathrm{h},
$$




$$
\mathrm{L}_{2}=\max \left\{\frac{\mathrm{h}}{1-\mathrm{h} / 2}, \frac{\mathrm{h}}{1-\mathrm{h} / 2}\right\}=\frac{\mathrm{h}}{1-\mathrm{h} / 2} .
$$

Thus, in all cases,

$$
\begin{aligned}
d(T x, T y) & \leq L_{1} d(x, y)+L_{2} d(x, T x) \\
& =h d(x, y)+\left(\frac{h}{1-h / 2}\right) d(x, T x)
\end{aligned}
$$

holds for all $x, y \in C$.

Also from (15) with $\mathfrak{y}=\mathfrak{u}=\mathrm{Tu}$, we have

$$
\begin{aligned}
d(T x, u) & \leq h \max \left\{d(x, u), \frac{d(x, T x)}{2}, \frac{d(x, u)+d(u, T x)}{2}\right\} \\
& \leq h \max \left\{d(x, u), \frac{d(x, T x)}{2}, \frac{d(x, u)+d(u, T x)}{2}\right\} \\
& \leq h \max \left\{d(x, u), \frac{d(x, u)+d(u, T x)}{2}, \frac{d(x, u)+d(u, T x)}{2}\right\} .
\end{aligned}
$$

Since for non-negative real numbers $a$ and $b$, we have

$$
\frac{a+b}{2} \leq \max \{a, b\} \text {. }
$$

Using (20) in (19), we have

$$
d(T x, u) \leq h d(x, u) .
$$

Now (21) gives

$$
\begin{aligned}
& d\left(T x_{n}, u\right) \leq h d\left(x_{n}, u\right) \\
& d\left(T y_{n}, u\right) \leq h d\left(y_{n}, u\right)
\end{aligned}
$$

and

$$
\mathrm{d}\left(T z_{n}, u\right) \leq h d\left(z_{n}, u\right) .
$$

Using (10), (21) and Lemma 1(ii), we have

$$
\begin{aligned}
\mathrm{d}\left(z_{n}, \mathfrak{u}\right) & =\mathrm{d}\left(\left(1-c_{n}\right) x_{n} \oplus c_{n} T x_{n}, u\right) \\
& \leq\left(1-c_{n}\right) d\left(x_{n}, u\right)+c_{n} d\left(T x_{n}, u\right) \\
& \leq\left(1-c_{n}\right) d\left(x_{n}, u\right)+c_{n} h d\left(x_{n}, u\right) \\
& =\left[1-(1-h) c_{n}\right] d\left(x_{n}, u\right) .
\end{aligned}
$$


Again using (10), (24), (25) and Lemma 1(ii), we have

$$
\begin{aligned}
d\left(y_{n}, u\right) & =d\left(\left(1-b_{n}\right) z_{n} \oplus b_{n} T z_{n}, u\right) \\
& \leq\left(1-b_{n}\right) d\left(z_{n}, u\right)+b_{n} d\left(T z_{n}, u\right) \\
& \leq\left(1-b_{n}\right) d\left(z_{n}, u\right)+b_{n} h d\left(z_{n}, u\right) \\
& =\left[\left(1-(1-h) b_{n}\right)\right] d\left(z_{n}, u\right) \\
& \leq\left[\left(1-(1-h) b_{n}\right)\right]\left[\left(1-(1-h) c_{n}\right)\right] d\left(x_{n}, u\right) \\
& \leq\left[1-(1-h) b_{n}\right] d\left(x_{n}, u\right) .
\end{aligned}
$$

Now using (10), (22), (23), (26) and Lemma 1(ii), we have

$$
\begin{aligned}
d\left(x_{n+1}, u\right) & =d\left(\left(1-a_{n}\right) T x_{n} \oplus a_{n} T y_{n}, u\right) \\
& \leq\left(1-a_{n}\right) d\left(T x_{n}, u\right)+a_{n} d\left(T y_{n}, u\right) \\
& \leq\left(1-a_{n}\right) h d\left(x_{n}, u\right)+a_{n} h d\left(y_{n}, u\right) \\
& \leq\left(1-a_{n}\right) h d\left(x_{n}, u\right)+a_{n} h\left[1-(1-h) b_{n}\right] d\left(x_{n}, u\right) \\
& =\left[\left(1-a_{n}\right) h+a_{n} h\left(1-(1-h) b_{n}\right)\right] d\left(x_{n}, u\right) \\
& =h\left[1-(1-h) a_{n} b_{n}\right] d\left(x_{n}, u\right) \\
& \leq\left[1-(1-h) a_{n} b_{n}\right] d\left(x_{n}, u\right) \\
& =\left(1-g_{n}\right) d\left(x_{n}, u\right)
\end{aligned}
$$

where $g_{n}=(1-h) a_{n} b_{n}$, since $0<h<1$ and by assumption of the theorem $\sum_{n=1}^{\infty} a_{n} b_{n}=\infty$, it follows that $\sum_{n=1}^{\infty} g_{n}=\infty$, therefore by Lemma 2, we get that $\lim _{n \rightarrow \infty} d\left(x_{n}, u\right)=0$. Thus $\left\{x_{n}\right\}$ converges strongly to a fixed point of $T$.

To show uniqueness of the fixed point $u$, assume that $w_{1}, w_{2} \in F(T)$ and $w_{1} \neq w_{2}$.

Applying (15) and using the fact that $0<\mathrm{h}<1$, we obtain

$$
\begin{aligned}
& \mathrm{d}\left(w_{1}, w_{2}\right)=d\left(T w_{1}, T w_{2}\right) \\
&=h \max \left\{d\left(w_{1}, w_{2}\right), \frac{d\left(w_{1}, T w_{1}\right)+d\left(w_{2}, T w_{2}\right)}{2},\right. \\
&=h \max \left\{d\left(w_{1}, w_{2}\right), \frac{d\left(w_{1}, w_{1}\right)+d\left(w_{2}\right)+d\left(w_{2}, T w_{1}\right)}{2}\right\} \\
&\left.\frac{d\left(w_{1}, w_{2}\right)+d\left(w_{2}, w_{1}\right)}{2}\right\}
\end{aligned}
$$




$$
\begin{aligned}
& =h \max \left\{\mathrm{d}\left(w_{1}, w_{2}\right), 0, \mathrm{~d}\left(w_{1}, w_{2}\right)\right\} \\
& \leq \mathrm{hd}\left(w_{1}, w_{2}\right) \\
& <\mathrm{d}\left(w_{1}, w_{2}\right), \text { since } 0<\mathrm{h}<1,
\end{aligned}
$$

which is a contradiction. Therefore $w_{1}=w_{2}$. Thus $\left\{x_{n}\right\}$ converges strongly to the unique fixed point of $\mathrm{T}$. This completes the proof.

Theorem 2 Let $\mathrm{C}$ be a nonempty closed convex subset of a complete CAT(0) space $\mathrm{X}$ and let $\mathrm{T}: \mathrm{C} \rightarrow \mathrm{C}$ be an operator satisfying the condition (15). Let $\left\{x_{n}\right\}$ be defined by the iteration scheme (11). If $\sum_{n=1}^{\infty} a_{n} b_{n}=\infty$, then $\left\{x_{n}\right\}$ converges strongly to the unique fixed point of $\mathrm{T}$.

Proof. The proof of Theorem 2 immediately follows from Theorem 1 by taking $c_{n}=0$ for all $n \geq 1$. This completes the proof.

The contraction condition (12) makes $T$ continuous function on $X$ while this is not the case with contractive conditions (13), (14) and (18).

The contractive conditions (13) and (14) both included in the class of Zamfirescu operators and so their convergence theorems for iteration process (10) are obtained in Theorem 1 in the setting of CAT(0) space.

Remark 1 Our result extends the corresponding result of [30] to the case of three-step iteration process (10) and from uniformly convex Banach space to the setting of $C A T(0)$ spaces.

Remark 2 Theorem 1 also extends Theorem B to the case of three-step iteration process (10) and from normed space to the setting of CAT(0) spaces.

\section{Application to contraction of integral type}

Theorem 3 Let $\mathrm{C}$ be a nonempty closed convex subset of a complete CAT(0) space $\mathrm{X}$ and let $\mathrm{T}: \mathrm{C} \rightarrow \mathrm{C}$ be an operator satisfying the following condition:

$$
\int_{0}^{d(T x, T y)} \mu(t) d t \leq h \int_{0}^{\max \left\{d(x, y), \frac{d(x, T x)+d(y, T y)}{2}, \frac{d(x, T y)+d(y, T x)}{2}\right\}} \mu(t) d t
$$

for all $\mathrm{x}, \mathrm{y} \in \mathrm{X}$ and $\mathrm{0}<\mathrm{h}<1$, where $\mu:[0,+\infty) \rightarrow[0,+\infty)$ is a Lebesgueintegrable mapping which is summable (i.e. with finite integral) on each compact subset of $[0,+\infty)$, nonnegative, and such that for each $\varepsilon>0, \int_{0}^{\varepsilon} \mu(t) d t>$ 0 . Let $\left\{x_{n}\right\}$ be defined by the iteration process (10). If $\sum_{n=1}^{\infty} a_{n} b_{n}=\infty$, then $\left\{x_{n}\right\}$ converges strongly to the unique fixed point of $\mathrm{T}$. 
Proof. The proof of Theorem 3 follows from Theorem 1 by taking $\mu(t)=1$ over $[0,+\infty)$ since the contractive condition of integral type transforms into a general contractive condition (15) not involving integrals. This completes the proof.

Example 1 Let $X=\{0,1,2,3,4,5\}$ and $\mathrm{d}$ be the usual metric of reals. Let $\mathrm{T}: \mathrm{X} \rightarrow \mathrm{X}$ be given by

$$
\left\{\begin{array}{rlr}
\mathrm{T}(\mathrm{x}) & =5, & \text { if } \quad x=0 \\
=3, & \text { otherwise. }
\end{array}\right.
$$

Again let $\mu:[0,+\infty) \rightarrow[0,+\infty)$ be given by $\mu(t)=1$ for all $\mathrm{t} \in[0,+\infty)$. Then $\mu:[0,+\infty) \rightarrow[0,+\infty)$ is a Lebesgue-integrable mapping which is summable (i.e. with finite integral) on each compact subset of $[0,+\infty)$, nonnegative, and such that for each $\varepsilon>0, \int_{0}^{\varepsilon} \mu(t) d t>0$.

Let us take $\mathrm{x}=0, \mathrm{y}=1$. Then from condition (28), we have

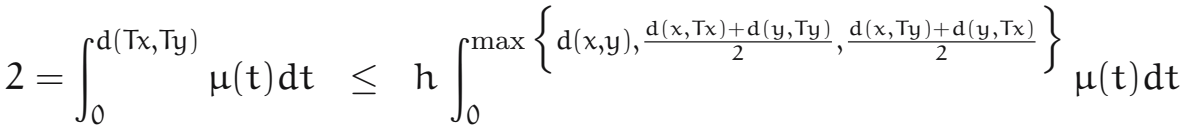

$$
\begin{aligned}
& =\mathrm{h} \max \left\{1, \frac{7}{2}, \frac{7}{2}\right\}
\end{aligned}
$$

which implies $\mathrm{h} \geq \frac{4}{7}$. Now if we take $0<\mathrm{h}<1$, then condition (28) is satisfied and 3 is of course a unique fixed point of $\mathrm{T}$.

The following corollaries are special cases of Theorem 3.

Corollary 1 Let $\mathrm{C}$ be a nonempty closed convex subset of a complete CAT(0) space $\mathrm{X}$ and let $\mathrm{T}: \mathrm{C} \rightarrow \mathrm{C}$ be an operator satisfying the following condition:

$$
\int_{0}^{d(T x, T y)} \mu(t) d t \leq h \int_{0}^{d(x, y)} \mu(t) d t
$$

for all $\mathrm{x}, \mathrm{y} \in \mathrm{X}$ and $\mathrm{h} \in(0,1)$, where $\mu:[0,+\infty) \rightarrow[0,+\infty)$ is a Lebesgueintegrable mapping which is summable (i.e. with finite integral) on each compact subset of $[0,+\infty)$, nonnegative, and such that for each $\varepsilon>0, \int_{0}^{\varepsilon} \mu(t) d t>$ 0. Let $\left\{x_{n}\right\}$ be defined by the iteration process (10). If $\sum_{n=1}^{\infty} a_{n} b_{n}=\infty$, then $\left\{x_{n}\right\}$ converges strongly to the unique fixed point of $\mathrm{T}$. 
Condition (29) is called Branciari [7] contractive condition of integral type.

Putting $\mu(t)=1$ in the condition (29), we get Banach contraction condition.

Proof. The proof of corollary 1 immediately follows from Theorem 1 by taking $\mu(t)=1$ over $[0,+\infty)$ and

$$
\max \left\{d(x, y), \frac{d(x, T x)+d(y, T y)}{2}, \frac{d(x, T y)+d(y, T x)}{2}\right\}=d(x, y)
$$

since the contractive condition of integral type transforms into a general contractive condition (12) not involving integrals. This completes the proof.

Corollary 2 Let $\mathrm{C}$ be a nonempty closed convex subset of a complete CAT(0) space $\mathrm{X}$ and let $\mathrm{T}: \mathrm{C} \rightarrow \mathrm{C}$ be an operator satisfying the following condition:

$$
\int_{0}^{d(T x, T y)} \mu(t) d t \leq b \int_{0}^{[d(x, T x)+d(y, T y)]} \mu(t) d t
$$

for all $\mathrm{x}, \mathrm{y} \in \mathrm{X}$ and $\mathrm{b} \in\left(0, \frac{1}{2}\right)$, where $\mu:[0,+\infty) \rightarrow[0,+\infty)$ is a Lebesgueintegrable mapping which is summable (i.e. with finite integral) on each compact subset of $[0,+\infty)$, nonnegative, and such that for each $\varepsilon>0, \int_{0}^{\varepsilon} \mu(t) d t>$ 0 . Let $\left\{x_{n}\right\}$ be defined by the iteration process (10). If $\sum_{n=1}^{\infty} a_{n} b_{n}=\infty$, then $\left\{x_{n}\right\}$ converges strongly to the unique fixed point of $\mathrm{T}$.

Condition (30) is called Kannan contractive condition [18] of integral type.

Putting $\mu(t)=1$ in the condition (30), we get Kannan contraction condition.

Proof. The proof of corollary 2 immediately follows from Theorem 1 by taking $\mu(t)=1$ over $[0,+\infty)$ and

$$
\max \left\{d(x, y), \frac{d(x, T x)+d(y, T y)}{2}, \frac{d(x, T y)+d(y, T x)}{2}\right\}=\frac{d(x, T x)+d(y, T y)}{2}
$$

since the contractive condition of integral type transforms into a general contractive condition (13) not involving integrals. This completes the proof.

Corollary 3 Let $\mathrm{C}$ be a nonempty closed convex subset of a complete $C A T(0)$ space $\mathrm{X}$ and let $\mathrm{T}: \mathrm{C} \rightarrow \mathrm{C}$ be an operator satisfying the following condition:

$$
\int_{0}^{d(T x, T y)} \mu(t) d t \leq c \int_{0}^{[d(x, T y)+d(y, T x)]} \mu(t) d t
$$


for all $x, y \in X$ and $c \in\left(0, \frac{1}{2}\right)$, where $\mu:[0,+\infty) \rightarrow[0,+\infty)$ is a Lebesgueintegrable mapping which is summable (i.e. with finite integral) on each compact subset of $[0,+\infty)$, nonnegative, and such that for each $\varepsilon>0, \int_{0}^{\varepsilon} \mu(t) d t>$ 0 . Let $\left\{x_{n}\right\}$ be defined by the iteration process (10). If $\sum_{n=1}^{\infty} a_{n} b_{n}=\infty$, then $\left\{x_{n}\right\}$ converges strongly to the unique fixed point of $\mathrm{T}$.

Condition (31) is called Chatterjae contractive condition [12] of integral type.

Putting $\mu(t)=1$ in the condition (31), we get Chatterjae contraction condition.

Proof. The proof of corollary 3 immediately follows from Theorem 1 by taking $\mu(t)=1$ over $[0,+\infty)$ and

$$
\max \left\{d(x, y), \frac{d(x, T x)+d(y, T y)}{2}, \frac{d(x, T y)+d(y, T x)}{2}\right\}=\frac{d(x, T y)+d(y, T x)}{2}
$$

since the contractive condition of integral type transforms into a general contractive condition (14) not involving integrals. This completes the proof.

Now, we give the examples in support of above corollaries.

Example 2 Let $\mathrm{X}$ be the real line with the usual metric $\mathrm{d}$ and suppose $\mathrm{C}=$ $[0,1]$. Define $\mathrm{T}: \mathrm{C} \rightarrow \mathrm{C}$ by $\mathrm{T}(\mathrm{x})=\frac{\mathrm{x}+1}{2}$ for all $\mathrm{x} \in \mathrm{C}$. Obviously $\mathrm{T}$ is selfmapping with a unique fixed point 1 . Again let $\mu:[0,+\infty) \rightarrow[0,+\infty)$ be given by $\mu(\mathrm{t})=1$ for all $\mathrm{t} \in[0,+\infty)$. Then $\mu:[0,+\infty) \rightarrow[0,+\infty)$ is a Lebesgue-integrable mapping which is summable (i.e. with finite integral) on each compact subset of $[0,+\infty)$, nonnegative, and such that for each $\varepsilon>0$, $\int_{0}^{\varepsilon} \mu(t) d t>0$.

If $x, y \in[0,1]$, then we have

$$
\mathrm{d}(\mathrm{T} x, \mathrm{Ty})=\left|\frac{x-y}{2}\right|
$$

Let us take $\mathrm{x}=0, \mathrm{y}=1$. Then from condition (29), we have

$$
\frac{1}{2}=\int_{0}^{d(T x, T y)} \mu(t) d t \leq h .1=h \int_{0}^{d(x, y)} \mu(t) d t
$$

which implies $\mathrm{h} \geq \frac{1}{2}$. Now if we take $0<\mathrm{h}<1$, then condition (29) is satisfied and 1 is of course a unique fixed point of $\mathrm{T}$. 
Example 3 Let $\mathrm{X}$ be the real line with the usual metric $\mathrm{d}$ and suppose $\mathrm{C}=$ $[0,1]$. Define $\mathrm{T}: \mathrm{C} \rightarrow \mathrm{C}$ by $\mathrm{T}(\mathrm{x})=\frac{\mathrm{x}}{4}$ for all $\mathrm{x} \in \mathrm{C}$. Obviously $\mathrm{T}$ is selfmapping with a unique fixed point 0 . Again let $\mu:[0,+\infty) \rightarrow[0,+\infty)$ be given by $\mu(\mathrm{t})=1$ for all $\mathrm{t} \in[0,+\infty)$. Then $\mu:[0,+\infty) \rightarrow[0,+\infty)$ is a Lebesgue-integrable mapping which is summable (i.e. with finite integral) on each compact subset of $[0,+\infty)$, nonnegative, and such that for each $\varepsilon>0$, $\int_{0}^{\varepsilon} \mu(t) d t>0$.

If $x, y \in[0,1]$, then we have

$$
\mathrm{d}(\mathrm{Tx}, \mathrm{Ty})=\left|\frac{x-y}{4}\right|
$$

Let us take $\mathrm{x}=0, \mathrm{y}=1$. Then from condition (30), we have

$$
\frac{1}{4}=\int_{0}^{d(T x, T y)} \mu(t) d t \leq b \cdot \frac{3}{4}=b \int_{0}^{[d(x, T x)+d(y, T y)]} \mu(t) d t
$$

which implies $\mathrm{b} \geq \frac{1}{3}$. Now if we take $0<\mathrm{b}<\frac{1}{2}$, then condition (30) is satisfied and 0 is of course a unique fixed point of $\mathrm{T}$.

Example 4 Let $\mathrm{X}$ be the real line with the usual metric $\mathrm{d}$ and suppose $\mathrm{C}=$ $[0,1]$. Define $\mathrm{T}: \mathrm{C} \rightarrow \mathrm{C}$ by $\mathrm{T}(\mathrm{x})=\frac{\mathrm{x}}{4}$ for all $\mathrm{x} \in \mathrm{C}$. Obviously $\mathrm{T}$ is self-mapping with a unique fixed point 0 . Again let $\mu:[0,+\infty) \rightarrow[0,+\infty)$ be given by $\mu(\mathrm{t})=$ 1 for all $\mathrm{t} \in[0,+\infty)$. Then $\mu:[0,+\infty) \rightarrow[0,+\infty)$ is a Lebesgue-integrable mapping which is summable (i.e. with finite integral) on each compact subset of $[0,+\infty)$, nonnegative, and such that for each $\varepsilon>0, \int_{0}^{\varepsilon} \mu(t) d t>0$.

If $x, y \in[0,1]$, then we have

$$
\mathrm{d}(\mathrm{T} x, \mathrm{Ty})=\left|\frac{x-y}{4}\right|
$$

Let us take $\mathrm{x}=0, \mathrm{y}=1$. Then from condition (31), we have

$$
\frac{1}{4}=\int_{0}^{d(T x, T y)} \mu(t) d t \leq c \cdot \frac{5}{4}=c \int_{0}^{[d(x, T y)+d(y, T x)]} \mu(t) d t
$$

which implies $\mathrm{c} \geq \frac{1}{5}$. Now if we take $0<\mathrm{c}<\frac{1}{2}$, then condition (31) is satisfied and 0 is of course a unique fixed point of $\mathrm{T}$. 


\section{Conclusion}

The Ciric quasi contractive operator [10] is more general than Banach contraction, Kannan contraction, Chatterjea contraction and Zamfirescu operators. Thus the results obtained in this paper are improvement and generalization of several known results from the existing literature (see, e.g., [4, 30] and some others).

\section{Acknowledgements}

The author would like to thanks the anonymous referee for his careful reading and valuable suggestions on the manuscript.

\section{References}

[1] M. Abbas, T. Nazir, A new faster iteration process applied to constrained minimization feasibility problems, Mat. Vesnik, 66 (2) (2014), 223-234

[2] R. P. Agarwal, Donal O'Regan and D.R. Sahu, Iterative construction of fixed points of nearly asymptotically nonexpansive mappings, Nonlinear Convex Anal., 8 (1) (2007), 61-79.

[3] A. Abkar, M. Eslamian, Common fixed point results in CAT(0) spaces, Nonlinear Anal.: TMA, 74 (5) (2011), 1835-1840.

[4] V. Berinde, Iterative approximation of fixed points, Baia Mare: Efemeride, 2000 .

[5] V. Berinde, Picard iteration converges faster than Mann iteration for a class of quasi contractive operators, Fixed Point Theory Appl., 2 (2004), 97-105.

[6] V. Berinde, Iterative approximation of fixed points, Springer-Verlag, Berlin Heidelberg, 2007.

[7] A. Branciari, A fixed point theorem for mappings satisfying a general contractive condition of integral type, Int. J. Math. Math. Sci., 29 (2002), $531-536$.

[8] M. R. Bridson, A. Haefliger, Metric spaces of non-positive curvature, Vol. 319 of Grundlehren der Mathematischen Wissenschaften, Springer, Berlin, Germany, 1999. 
[9] F. Bruhat, J. Tits, "Groups reductifs sur un corps local", Institut des Hautes Etudes Scientifiques. Publications Mathematiques, 41 (1972), 5251.

[10] L. B. Ciric, A generalization of Banach principle, Proc. Amer. Math. Soc., 45 (1974), 727-730.

[11] P. Chaoha and A. Phon-on, A note on fixed point sets in CAT(0) spaces, J. Math. Anal. Appl., 320 (2) (2006), 983-987.

[12] S. K. Chatterjae, Fixed point theorems compactes, Rend. Acad. Bulgare Sci., 25 (1972), 727-730.

[13] S. Dhompongsa, A. Kaewkho, B. Panyanak, Lim's theorems for multivalued mappings in CAT(0) spaces, J. Math. Anal. Appl., 312 (2) (2005), 478-487.

[14] S. Dhompongsa, B. Panyanak, On $\triangle$-convergence theorem in CAT(0) spaces, Comput. Math. Appl., 56 (10) (2008), 2572-2579.

[15] R. Espinola, A. Fernandez-Leon, CAT(k)-spaces, weak convergence and fixed point, J. Math. Anal. Appl., 353 (1) (2009), 410-427.

[16] N. Hussain, M.A. Khamsi, On asymptotic pointwise contractions in metric spaces, Nonlinear Anal.: TMA 71, (10) (2009), 4423-4429.

[17] S. Ishikawa, Fixed points by a new iteration method, Proc. Amer. Math. Soc., 44 (1974), 147-150.

[18] R. Kannan, Some results on fixed point theorems, Bull. Calcutta Math. Soc., 60 (1969), 71-78.

[19] M. A. Khamsi, W. A. Kirk, An introduction to metric spaces and fixed point theory, Pure Appl. Math, Wiley-Interscience, New York, NY, USA, 2001.

[20] S. H. Khan, M. Abbas, Strong and $\triangle$-convergence of some iterative schemes in CAT(0) spaces, Comput. Math. Appl., 61 (1) (2011), 109116.

[21] A. R. Khan, M. A. Khamsi, H. Fukhar-ud-din, Strong convergence of a general iteration scheme in CAT(0) spaces, Nonlinear Anal.: TMA, $\mathbf{7 4}$ (3) (2011), 783-791. 
[22] W. A. Kirk, Geodesic geometry and fixed point theory, in Seminar of Mathematical Analysis (Malaga/Seville, 2002/2003), Vol. 64 of Coleccion Abierta, 195-225, University of Seville Secretary of Publications, Seville, Spain, 2003.

[23] W. A. Kirk, Geodesic geometry and fixed point theory II, in International Conference on Fixed point Theory and Applications, 113-142, Yokohama Publishers, Yokohama, Japan, 2004.

[24] W. Laowang, B. Panyanak, Strong and $\triangle$ convergence theorems for multivalued mappings in CAT(0) spaces, J. Inequal. Appl., Vol. 2009, Article ID 730132, 16 pages, 2009.

[25] L. Leustean, A quadratic rate of asymptotic regularity for CAT(0)-spaces, J. Math. Anal. Appl., 325 (1) (2007), 386-399.

[26] W. R. Mann, Mean value methods in iteration, Proc. Amer. Math. Soc., 4 (1953), 506-510.

[27] Y. Niwongsa, B. Panyanak, Noor iterations for asymptotically nonexpansive mappings in CAT(0) spaces, Int. J. Math. Anal., 4 (13) (2010), 645-656.

[28] M. A. Noor, New approximation schemes for general variational inequalities, J. Math. Anal. Appl., 251 (1) (2000), 217-229.

[29] A. Rafiq, Fixed points of Ciric quasi-contractive operators in generalized convex metric spaces, General Math., 14 (3) (2006), 79-90.

[30] B. E. Rhoades, Fixed point iteration using infinite matrices, Trans. Amer. Math. Soc., 196 (1974), 161-176.

[31] S. Saejung, Halpern's iteration in CAT(0) spaces, Fixed Point Theory Appl., Vol. 2010, Article ID 471781, 13 pages, 2010.

[32] N. Shahzad, Fixed point results for multimaps in CAT(0) spaces, Topology and its Applications, 156 (5) (2009), 997-1001.

[33] D. Thakur, B. S. Thakur, M. Postolache, New iteration scheme for numerical reckoning fixed points of nonexpansive mappings, J. Ineq. Appl., 2014:328, 2014.

[34] T. Zamfirescu, Fixed point theorems in metric space, Arch. Math. (Basel), 23 (1972), 292-298. 